\title{
Comparative study of intraperitoneal adhesions related to light-weight polypropylene mesh and type I polymerized and purified bovine collagen coated light-weight polypropylene mesh in rabbits ${ }^{1}$
}

Diego Paim Carvalho Garcia', Clarissa Santos Neto", Cristiana Buzelin Nunes"I, Marcelo Araújo Buzelin' $^{\prime V}$, Andy Petroianuv, Luiza Ohasi de Figueiredo ${ }^{V \prime}$, Andreia Souto da MottaVII, Cristiane de Barros Gaspar ${ }^{\mathrm{VII}}$, Luiz Ronaldo Alberti ${ }^{\mathrm{VIII}}$

'PhD, Associate Professor, Department of Surgery, Instituto de Ensino e Pesquisa da Santa Casa, Belo Horizonte-MG, Brazil. Intellectual and scientific content of the study, design the protocol, technical procedures, manuscript writing.

"MD, Hospital Universitário São José, Belo Horizonte-MG, Brazil. Acquisition and interpretation of data, statistical analysis, design the protocol, technical procedures, macroscopic and histopathological examinations.

I'PhD, Assistant Professor, Department of Pathology, School of Medicine, Universidade Federal de Minas Gerais (UFMG), Belo Horizonte-MG, Brazil. Technical procedures, macroscopic and histopathological examinations, interpretation of data. IVBiologist, Master in Pathology, Instituto de Ensino e Pesquisa da Santa Casa, Belo Horizonte-MG, Brazil. Technical procedures, macroscopic and histopathological examinations, interpretation of data.

${ }^{\mathrm{V}} \mathrm{PhD}$, Full Professor, Department of Surgery, School of Medicine, UFMG, Belo Horizonte-MG, Brazil. Intellectual and scientific content of the study, design the protocol, provided guidelines for the surgical interventions, supervised all phases of the study.

V'MD, Hospital Felício Rocho, and Fellow of Oncologic Surgery, Hospital Alberto Cavalcanti, Belo Horizonte-MG, Brazil. Acquisition and interpretation of data, statistical analysis, manuscript preparation.

VIIMD, Fellow of Plastic Surgery, Hospital Felício Rocho, Belo Horizonte-MG, Brazil Acquisition and interpretation of data, statistical analysis, manuscript preparation.

VIIIPhD, Associate Professor, Department of Surgery, School of Medicine, UFMG, and Instituto de Ensino e Pesquisa da Santa Casa, Belo Horizonte-MG, Brazil. Acquisition and interpretation of data, statistical analysis, design the protocol, technical procedures.

\section{Abstract}

Purpose: To compare the effectiveness of light-weight polypropylene mesh coated with polymerized and purified bovine type I collagen (Surgidry HNB) in the treatment of abdominal wall defect and the degree of adhesion formation.

Methods: Two types of polypropylene mesh were implanted after creation of defect measuring $6.0 \mathrm{~cm} \times 5.5 \mathrm{~cm}$ in the anterior abdominal wall of 32 male New Zealand breed rabbits, divided in two groups $(n=32)$ : (1) light-weigh macroporous polypropylene, $(2)$ type I polymerized and purified bovine collagen coated light-weigh macroporous polypropylene. These animals were further accessed for adhesions, histological evaluation of inflammation and wall's thickness.

Results: The percentage of the area adhered in group $1(62.31 \pm 16.6)$ was higher compared to group $2(22.19 \pm 14.57)(p<0.05)$. There was an association between the percentage of the covered area by adhesions and the type of adhesion, toughness and the scores obtained by the adhesion score by correlation analysis $(p<0.05)$. There was no difference between the groups in any variables in relation to the degree of inflammation.

Conclusion: The purified type I bovine collagen coated light-weigh polypropylene mesh showed to be effective in the repair of abdominal wall defects and reducing adhesion formation. Key words: Incisional Hernia. Surgical Mesh. Polypropylenes. Collagen Type I. Rabbits. 


\section{- Introduction}

The popularization of laparascopic correction of incisonal hernias with intraperitonial fixation of mesh raised the concerns towards the formation of postsurgical adhesions. The lack of and "ideal" mesh lead to the development of a series of composed meshes, mixing different types of materials ${ }^{1-4}$. The main advantage of the composed meshes it is the possibility of intraperitoneal placement, with minimal adhesion formation. Regardless of the large spectrum of brand options available, almost all manufacturers still use the same three basic materials - polypropylene, polyester or polytetrafluoroethylene (PTFE). These materials are used in combination one with another or with a variety of different materials, such as titanium, omega 3, monocryl, polyvinylidene-fluoride (PVDF) and hyaluronate ${ }^{2-4}$.

Every mesh may produce adhesions once put in contact with the intestine, but the extension of the adherence is determined by the width of its pores, filament structure and the area of the surface in contact with the bowels ${ }^{1,2,4}$. Standart meshes or heavy-weight tend to produce a rather intense fibrotic tissue response, granting its adhesion to the abdominal wall, but, also, to the intrabdominal structures. Nevertheless, microporous meshes and PPTFE based meshes present with lower fibrotic tissue growth, decreasing the formation of adhesions, but at the same time reducing its ability to adhere to the abdominal wall ${ }^{2,3,6}$.

Those opposed findings illustrate the difficulty within the manufacturing of an ideal prosthesis that presents adequate adherence to the abdominal without compromising intraabdominalstructures. Composed meshes intent to provide an additional surface, that allows safe placement when in contact with intraabdominal structures due to the inflammatory reaction that induces the proliferation of peritoneum mesothelial cells over the mesh surface. The peritoneum regenerates itself over the mesh in 7 days, avoiding the formation of adhesions in the covered area ${ }^{1,3,5,6 .}$.

Studies that relate the treatment of abdominal wall defects to the formation of adhesions are mostly performed in animals and widely observed in medical literature ${ }^{5,6,8}$. The macroscopic quantification, due to the direct observation of the adhesions, makes studies in human beings ethically prohibitive, once a new surgical procedure would be necessary, allowing the formation of new adhesions. Therefore, studies performed in humans have the bias of being performed under the treatment of complications caused by initial repairs, such as hernia recurrence or intestinal obstruction caused by adhesions $s^{3,4,6,7}$.

The use of polymerized and purified type I bovine pericardium organic matrix for the correction of abdominal wall defects in rabbits was studied, and it was come to the conclusion that this material is not effective due to the high incidence of hernia formation. However, this material showed to be biocompatible, causing minimal inflammatory and foreign body reaction, having a better architectural arrangement of the collagen fibers, allowing its adequate use as a barrier against the formation of adhesions $s^{4-6,8}$.

In virtue of the absence of an "ideal" prosthesis, the material used must be individualized, taken each case into consideration. This study aims to bring a new association of materials to be used for the adequate treatment of the abdominal wall defects and reduction of formation of intrabdominal adhesions. The composed mesh produced for this study is the result of the combination of a polypropylene macroporous light-weight mesh - Repol Mesh - and a polymerized and purified type I bovine pericardium organic matrix sterilized with gama radiation, combined together with a Vycril ${ }^{\circ}$ 5-0 
suture. This composed mesh, under study, has never been described in medical literature so far.

\section{- Methods}

This work was carried out in accordance with that recommended by the International Standards for the Protection of Animals and the Brazilian Animal Experimentation Code (1988), and was approved by the Committee of Ethics in Animal Experimentation, Universidade Federal de Minas Gerais of the protocol number 099/2011.

Thirty-two New Zeland male rabbits, three months of age and with weights above two kilograms, acquired from the Experimental Veterinary Farm were studied. All of the rabbits were identified and placed in the Biothery of the School of Medicine, one animal per cage. They received daily rations for rabbits and filtered water ad libitum.

The rabbits were anesthetized with an intramuscular injection in the gluteal region with $5 \%$ ketamine hydrochloride (Ketamin- $\mathrm{S}^{\circ}(+)$, Cristália, Itapira-SP) at a dose of $35 \mathrm{mg} / \mathrm{kg}(0.7$ $\mathrm{ml} / \mathrm{kg})$, coupled with $2 \%$ xylazine hydrochloride (Rompun ${ }^{\circ}$, Bayer, Sao Paulo-SP) at a dose of 6 $\mathrm{mg} / \mathrm{kg}(0.3 \mathrm{ml} / \mathrm{kg})$. When necessary, half of the initial dose of the anesthesia was applied. During the entire period of anesthesia, the heart and respiration rate were observed, as were the rabbit's voluntary movements, in an attempt to detect complications.

After the trichotomy of the abdomen, antisepsis was carried out, using a $2 \%$ degerming chlorhexidine solution followed by a $70 \%$ alcohol solution and the setting of surgical fields. A defect measuring $6 \mathrm{~cm} \times 5.5 \mathrm{~cm}$ was created in the ventral abdominal wall with removal of a muscle-aponeurotic sheaf, using single card template for all animals. The rabbits were then divided into two groups of
16 animals by a random drawing $(n=32)$ for placement of the mesh in the intraperitoneal space:

- Group 1: placement of light-weigh macroporous polypropylene mesh, $8.0 \mathrm{~cm} x$ $7.5 \mathrm{~cm}$ on the borders of the abdominal wall within $1 \mathrm{~cm}$ of the edge of the mesh, with a 3-0 monofilament polypropylene suture.

- Group 2: placement of type I polymerizedand purified bovinecollagen coated light-weigh macroporous polypropylene, $8.0 \mathrm{~cm}$ $x 7.5 \mathrm{~cm}$ on the borders of the abdominal wall within $1 \mathrm{~cm}$ of the edge of the mesh using only the polypropylene layer as anchorage, with a 3-0 monofilament polypropylene suture.

After the surgery and during the entire follow-up period, the rabbits received rations and filtered water ad libitum, and were kept in individual cages, under appropriate conditions of hygiene, ventilation and natural illumination.

At the end of the follow-up period, on the 90th postoperative day, the animals were killed with inhalation of carbon dioxide in a closed chamber following an intramuscular injection of $2 \mathrm{ml}$ of xylazine $(10 \mathrm{mg} / \mathrm{kg}$ ).

A laparotomy in $U$, released a quadrangular portion of the abdominal wall, to study the occurrence of intrabdominal adhesions within evaluation of the following aspects: intrabdominal organs with adhesions, presence of vascularization, level of resistance, percentage of the mesh's surface covered by adhesions and incorporation of the mesh in the edges of wall defect.

The analyses of percentage of coverage by adhesions were calculated using the IMAGEJ software 1.47V (Wayne Rasband, National Institutes of Health, USA), and two images, taken with a Canon EOS $1100 D$ camera with EFD $18-55 \mathrm{~mm}$ lenses, of each animal. Mascroscopic evaluation of the adhesions were made using the adhesion score described by the Surgical Membrane Study Group ${ }^{9}$ (Table 1). 
Table 1 - Adhesion score of the Surgical Membrane Study Group.

\begin{tabular}{ll} 
Adhesion characteristics & Score \\
\hline Extent of site involvement (\%) & 0 \\
None & 1 \\
$<25 \%$ & 2 \\
$<50 \%$ & 3 \\
$<75 \%$ & 4 \\
$<100 \%$ & \\
& \\
Type & 0 \\
None & 1 \\
Filmy, transparent, avascular & 2 \\
Opaque, translucent, avascular & 3 \\
Opaque, capillaries present & 4 \\
Opaque, large vessels present & \\
& \\
Tenacity & \\
None & \\
Adhesions falls apart & 11 \\
Adhesions lysed with traction & 2 \\
Adhesions requiring sharp dissection & 3 \\
Possible total & \\
\hline
\end{tabular}

The removed abdominal wall was then prepared for histological study and stained with hematoxylin and eosin (HE) and Masson trichrome staining. The microscopic evaluation with HE and Masson's staining was performed to quantify the foreign body gigantic cells, granulomas, chronic inflammation, acute inflammation and neovascularization. A measurement of the wall thickness was also performed.

The analyses were performed using SPSS (Statistical Package for Social Science) version 20.0, 2012 of Microsoft Excel database tables. The variables were analyzed regarding their distribution and supposition of normality was verified by the Shapiro-Wild test. For the analysis of the qualitative variables Pearson's Chi-square distribution and Fisher's exact test were used, and the quantitative analyses were performed using Student's t test for the independent samples.

For the correlation analysis Pearson's coefficients were adopted in the cases in which the variables were not categorized. For the total punctuation score, categorized in low and high risk, the Biserial Correlation Coefficient, which is an estimate of Person's Linear Correlation Coefficient, was used.

\section{Results}

All of the animals recovered spontaneously from the surgeries and survived the three-month experiment and presented no late complications, such as hematomas, surgical wound infection, fistulas, incisional hernia relapse or mesh extrusion. Two animals, one from each group, presented surgical wound seroma, with spontaneous regression and no need of surgical approach.

\section{Adhesions}

An adequate integration of the mesh with the abdominal wall was observed in all animals. Within the composite mesh group it was observed adhesion of intrabdominal organs onto the side non covered by collagen in all animals, meanwhile the polypropylene mesh group presented adhesions to the entire surface of the mesh in all animals (Figure 1). 

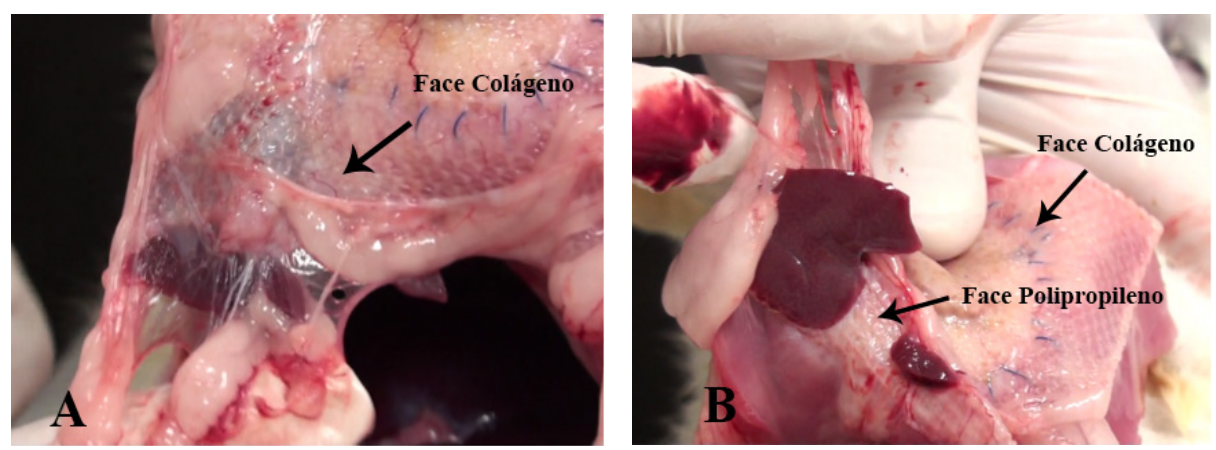

Figure 1 - A: Collagen side of composite mesh, facing visceral organs, without adhesions. B: Polypropylene side with visceral adhesions.

A rate of $56.25 \%$ of the animals belonging to the polypropylene mesh group presented intestinal adherence to the mesh, while none of the animals of composite mesh group presented the same type of adhesion, showing, statistically, considerable difference between this complication between both groups ( $p=0.0004)$ (Table 2$)$.

Table 2 - Type of adhesions according to adhered structure.

\begin{tabular}{lllll}
\hline Organs & $\begin{array}{l}\text { Polypropylene mesh } \\
\text { Visceral side } \\
\text { (Polypropylene) }\end{array}$ & $\begin{array}{l}\text { Parietal side } \\
\text { (Polypropylene) }\end{array}$ & $\begin{array}{l}\text { Composite mesh } \\
\text { Visceral side } \\
\text { (Collagen) }\end{array}$ & $\begin{array}{l}\text { Parietal side } \\
\text { (Polypropylene) }\end{array}$ \\
\hline Omentum & $9^{*}(56.25 \%)$ & $16(100 \%)$ & $6(37.5 \%)$ & $16(100 \%)$ \\
Small Bowel & $9(56.25 \%)^{* *}$ & $0(0 \%)$ & $0(0 \%)^{* *}$ & $4(18.75 \%)$ \\
Colon & $16(100 \%)^{* * *}$ & $0(0 \%)$ & $10(62.5 \%)^{* * *}$ & $0(0 \%)$ \\
Líver & $0(0 \%)$ & $9(56.25 \%)$ & $0(0 \%)$ & $9(56.25 \%)$ \\
\hline
\end{tabular}

${ }^{*} \mathrm{n}=16$ animals in each group; $*{ }^{*} \mathrm{p}=0.0004 ; * * * \mathrm{p}=0.007$

Area covered by adhesions

The percentage of the mesh's surface covered by adhesions was smaller in the composite mesh group when compared to the polypropylene group (63.31\% vs. $22.91 \%$; p<0.05) (Table 3).

Table 3 - Average percentage of area covered by adhesions.

\begin{tabular}{llll} 
Variant & Polypropylene mesh & Composite mesh & p \\
\hline $\begin{array}{l}\text { Percentage of area covered } \\
\text { by adhesions* }\end{array}$ & $62.31 \pm 16.6$ & $22.19 \pm 14.57$ & $<0.00^{* *}$ \\
\hline
\end{tabular}

*quantitative variant, represented as average \pm standart deviation; ${ }^{* *} t$ student test

Moreover, when analyzing both groups total scores according to the Adhesion Score of the Surgical Membrane study group, it was possible to observe that $93.75 \%$ of the animals belonging to polypropylene group presented with a total score above 5, however only $6.25 \%$ of the animals from the group that received the composite mesh presented a comparable score (Tables 4 and 5). 
Table 4 - Comparison of groups according to the adhesion score of the Surgical Membrane Study Group.

\begin{tabular}{|c|c|c|c|}
\hline $\begin{array}{l}\text { Adhesion characteristics } \\
\text { Extent of site involvement (\%) }\end{array}$ & Polypropylene mesh N(\%) & Composite mesh $\mathrm{N}(\%)$ & $\mathbf{P}$ \\
\hline None - 0 & $0(0.0 \%)$ & $0(0.0 \%)$ & - \\
\hline$<25 \%-1$ & $2(12.5 \%)$ & $14(87.5 \%)$ & 0.02 \\
\hline$<50 \%-2$ & $6(37.5 \%)$ & $2(12.5 \%)$ & 0.10 \\
\hline$<75 \%-3$ & $6(37.5 \%)$ & $0(0.0 \%)$ & 0.02 \\
\hline$<100 \%-4$ & $2(12.5 \%)$ & $0(0.0 \%)$ & 0.04 \\
\hline \multicolumn{4}{|l|}{ Type } \\
\hline None - 0 & $0(0,0 \%)$ & $0(0,0 \%)$ & - \\
\hline Filmy, transparente, avascular - 1 & $1(6.3 \%)$ & $12(75 \%)$ & 0.01 \\
\hline Opaque, translucent, avascular - 2 & $3(18.8 \%)$ & $3(18.8 \%)$ & - \\
\hline Opaque, capillaries present -3 & $11(68.8 \%)$ & $0(0.0 \%)$ & 0.01 \\
\hline Opaque, larger vessels present -4 & $1(6.3 \%)$ & $1(6.3 \%)$ & - \\
\hline \multicolumn{4}{|l|}{ Tenacity } \\
\hline None -0 & $0(0.0 \%)$ & $0(0.0 \%)$ & - \\
\hline Adhesions falls apart -1 & $1(6.3 \%)$ & $10(62.5 \%)$ & 0.02 \\
\hline Adhesions lysed with traction -2 & $1(6.3 \%)$ & $5(31.3 \%)$ & 0.02 \\
\hline Adhesions requiring sharp dissection -3 & $14(87.5 \%)$ & $1(6.3 \%)$ & $<0.00$ \\
\hline
\end{tabular}

Table 5 - Total obtained on the Adhesion score by groups 1 and 2 .

\begin{tabular}{ccl}
\hline Total score & $\begin{array}{c}\text { Polypropylene } \\
\text { mesh N(\%) }\end{array}$ & $\begin{array}{l}\text { Composite } \\
\text { mesh N(\%) }\end{array}$ \\
\hline 1 & - & - \\
2 & - & - \\
3 & $1(6.25 \%)$ & $9(56.25 \%)$ \\
4 & - & $3(18.75 \%)$ \\
5 & - & $3(18.75 \%)$ \\
6 & $1(6.25 \%)$ & - \\
7 & $3(18.75 \%)$ & - \\
8 & $2(12.5 \%)$ & - \\
9 & $6(37.5 \%)$ & $1(6.25 \%)$ \\
10 & $1(6.25 \%)$ & - \\
11 & $1(6.25 \%)$ & - \\
Total of specimens & $16(100 \%)$ & $16(100 \%)$ \\
\hline
\end{tabular}

Level of adherences - Tenacity

According to the classification of adhesions, it was possible to observe that the groups presented different tenacity levels. From the polypropylene mesh group, 2 animals presented each levels 1 and 2 of adherences, giving a $6.3 \%$ for the respective categories. Meanwhile 14 animals presented level 3 adherences (87.5\%). Amongst the composite mesh group it was seen a larger occurrence of adhesions levels 1 and 2 ( 10 and 5 animals each, comprehending, statistically, $62.5 \%$ and $31,3 \%$ of the total) and a reduced number of level 3 adhesions, with only one animal affected (6,3\%) (Figure 2). 


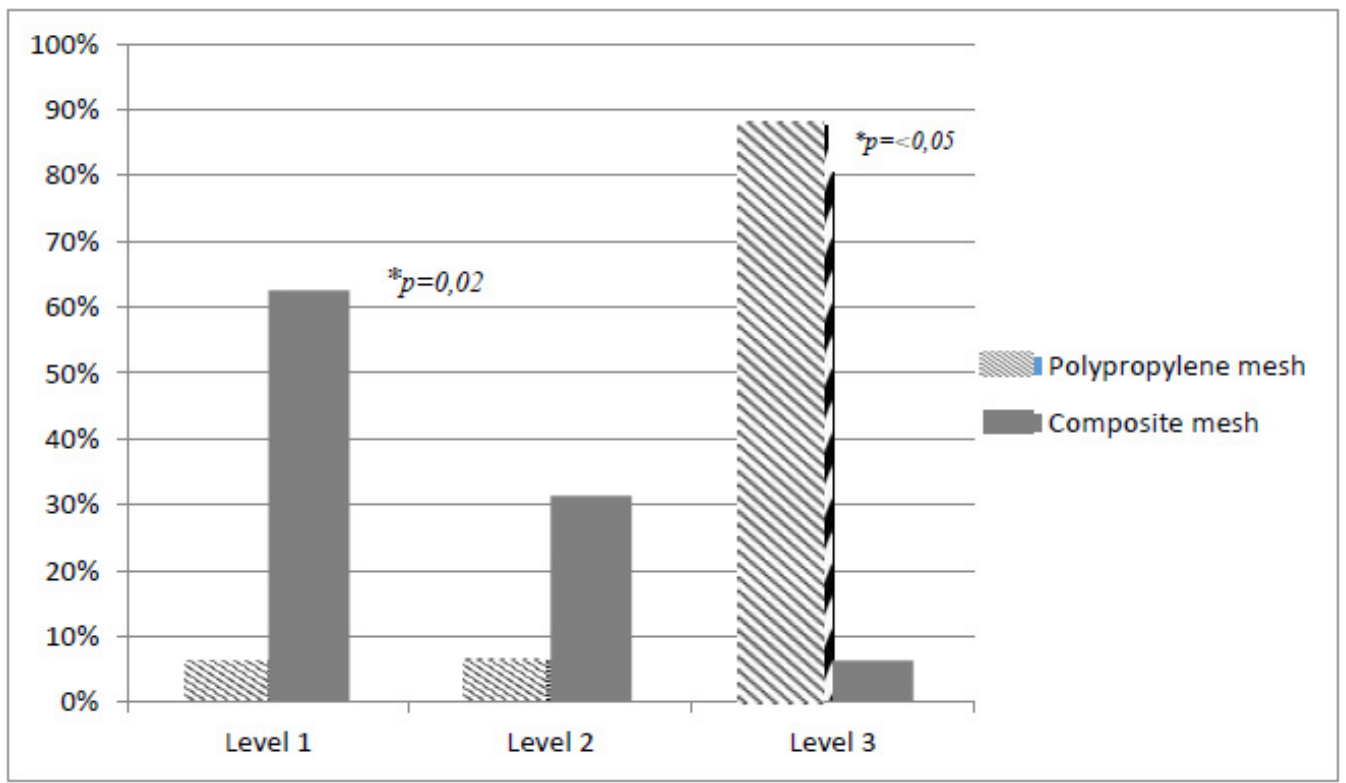

Figure 2 - Comparison of tenacity levels between both groups.

\section{Correlation analysis}

The correlation analysis was made between the average area of mesh covered by adhesions and the following variants: type of adhesions, tenacity and adherence score. The average of the percentage of the surface covered by adhesions (41.9\%), was used to distribute all animals $(n=32)$ within 2 groups: below and above the average.

The first correlation, between the tenacity and the percentage of mesh covered by adhesions, showed that the animals with adhesions of tenacity ranked 1 were the ones that had adhesions below the average. None of the animals presented a score 0 , confirming the correlation between the increase of adhesion surface and the tenacity score (Table 6).

Table 6 - Correlation analysis (Fisher's exact test) between tenacity and average surface of mesh covered by adhesions.

\begin{tabular}{llll} 
& \multicolumn{3}{l}{ Percentage of covered area according to average (41.9\%) } \\
& Below average & Above average & p \\
\hline Score $0-1$ & $56.3 \%(9)$ & $12.5 \%(2)$ & $0.02 *$ \\
Score $2-3$ & $43.8 \%(7)$ & $87.5 \%(14)$ & \\
Total & $100 \%(16)$ & $100 \%(16)$ & \\
\hline
\end{tabular}

The second correlation used the Chisquare test to associate the type of adhesion and with percentage of the mesh surface covered by the adhesions. In the same way, that the animals that presented a smaller coverage area by the adhesions were ranked score 1 , showing a direct relation between the increase of adhesion surface and the type of adhesion score (Table 7). 
Table 7 - Correlation analysis (Chi-square test) between type of adhesion and average surface of mesh covered by adhesions.

\begin{tabular}{llll} 
& \multicolumn{2}{l}{ Percentage of covered area according to average (41.9\%) } \\
& Below average & Above average & p \\
\hline Score $0-1$ & $68.8 \%(11)$ & $12.5 \%(2)$ & $<0.00^{*}$ \\
Score $2-3-4$ & $31.3 \%(5)$ & $87.5 \%(14)$ & \\
Total & $100 \%(16)$ & $100 \%(16)$ & \\
\hline
\end{tabular}

The third, and last correlation, was made between the total result obtained according to the score of adhesions and the percentage of the mesh surface covered by the adhesions. This last correlation proved that the increase in the surface covered by adhesions is directly related to a higher adhesion score (Table 8).

Table 8 - Correlation analysis (Chi-square test) between Adhesion score result and the average surface of mesh covered by adhesions.

\begin{tabular}{llll} 
& \multicolumn{2}{l}{ Percentage of covered area according to average (41.9\%) } \\
& Below average & Above average & P \\
\hline Score $0-4$ & $68.8 \%(11)$ & $12.5 \%(2)$ & $<0.00^{*}$ \\
Score $5-11$ & $31.3 \%(5)$ & $87.5 \%(14)$ & \\
Total & $100 \%(16)$ & $100 \%(16)$ & \\
\hline
\end{tabular}

Microscopic analysis of inflammation degree

There was no statistical difference between none of the evaluated variables, according to modified Hooker's classification: giant foreign body cells, granulomas, chronic infection, acute inflammation and neovascularization.

\section{- Discussion}

Large sized animals are the most adequate for the development of abdominal wall defect models. The advantaged seen in utilizing small animals, such as rats and rabbits, lies in the advantage of lower cost, small sized accommodations and more accessible materials ${ }^{10}$.

In this study this fact was taken into consideration, and this acknowledgment made the choice of rabbits suitable for the development of the study. The choice weighted the benefits of the viability of the surgical procedure, without recurring to special surgical equipment, and the smaller level of biological complexity regarding the surgical procedure itself and the post-operative care necessary for the amount of animals required ${ }^{10}$.

In the present study both meshes were placed in the abdominal cavity, according to the laparoscopic technique, having it fixated to the edges of the abdominal defects and with a $1 \mathrm{~cm}$ overlap, minimum (respecting Pascal's principle). The lightweight polypropylene meshes were effective for the repair of the abdominal wall due to the outcome of no incisional hernia being developed by any of the animals submitted to the application of this prosthesis. 
The composite mesh produced for this study was effective for the reduction of the area adhered do the surface of the mesh. This result was impaired only by the exposition of a certain amount of polypropylene into de cavity causing omental, intestinal and liver adherences. Ideally, the mesh would be completely covered by bovine collagen, however the high costs and technological complexity of the production did not justify the cost-benefit of the attempt, once no studies proved its full effectiveness until the present moment.

Type 1 bovine collagen was effective, even with a long observational period, in the reduction of extension and degree of adherences according to Jenkins classification. For the correlation analysis, the animals were sorted in a manner that the results showing no adhesions or adhesions with small clinical significance (level I) were in the same group, and in those animals the composite mesh showed a positive correlation in all variables evaluated. Therefore, the composite mesh, besides the reduction of adherence area, also presented looser adhesions.

As predicted, there was no statistical difference in any of the analyzed variables, according to Hooker's modified classification. The inflammatory process induced by the presence of the lightweight polypropylene mesh was the same in both groups, and the organic collagen layer acted as a barrier preventing the formation of intrabdominal adherences between the intrabdominal organs and the polypropylene mesh, modulating the biological response to the presence of the mesh, but without interfering on its efficiency on the repair of the abdominal defect and its resistance to the mechanic tensions of the abdominal wall.

\section{Conclusions}

The type I bovine collagen and lightweight polypropylene composite mesh is an effective prosthetic for the repair of abdominal wall defects associated with lesser adhesions, considering that there is no perfect model or manufactured mesh that suits the prerogative of not producing any foreign body reaction, according to modern literature.

Although the animal model utilized does not suits the requirements of extrapolating this study results for other biological models, such as humans, this experimental study is the first that evaluates and analyses both materials assembled and may influence the investment in advanced technology for the production of a polypropylene prosthetic mesh fully coated by bovine collagen, so that further studies might be produced.

\section{References}

1. Brown CN, Finch JG. Which mesh for hernia repair? Ann R Coll Surg Engl. 2010 May;92(4):272-8. PMID: 20501011.

2. Biondo-Simões $M L$, Carvalho $L B$, Conceição LT, Santos KB, Schiel WA, Arantes M, Silveira TD, Magri JC, Gomes FF. Comparative study of Polypropylene versus Parietex composite ${ }^{\circledR}$, Vicryl ${ }^{\circledR}$, and Ultrapro ${ }^{\circledR}$ meshes, regarding the formation of intraperitoneal adhesions. Acta Cir Bras. 2017 Feb;32(2):9107. PMID: 230076.

3. O'Dwyer, Kingsworth AN, Molloy RG, Small PK, Lammers B, Horeyseck G. Randomized clinical trial assessing impact of a lightweight or heavyweight mesh on chronic pain after inguinal hernia repair. Br J Surg. 2005 Feb;92(2):166-70. PMID: 15584057.

4. Vogels RR, van Barneveld KW, Bosmans JW, Beets G, Gijbels MJ, Schereinemacher MH, Bouvy ND. Long-term evaluation of adhesion formation and foreign body response to three new meshes. Surg Endosc. 2015 Aug;29:2251-9. PMID: 25361655.

5. Schreinemacher MH, Emans PJ, Gijbels MJ, Greve JW, Beets GL, Bouvy ND. Degradation of mesh coatings and intraperitoneal adhesion formation in an experimental model. Br J Surg. 2009 Mar;96(3):305-13. PMID: 19224521.

6. Schreinemacher $\mathrm{MH}$, van Barneveld $\mathrm{KW}$, Dikmans RE, Gijbels MJ, Greve JW, Bouvy 
ND. Coated meshes for hernia repair provide comparable intraperitoneal adhesion prevention. Surg Endosc. 2013 Nov;27(11):4202-9. PMID: 23749270.

7. Garcia DP, Santos C Neto, Hubner PN, Furtado TA, Petroianu A, Figueiredo LO, Alberti LR. Treatment of abdominal wall hernia with suture, or polypropylene, or collagen prostesis. Acta Cir Bras. 2016 Jun;31(6):371-6. PMID: 27355743.

8. Klosterhalfen $B$, Junge $K$, Klinge $U$. The lightweight and large porous mesh concept for hernia repair. Expert Rev Med Devices. 2005 Jan;2(1):103-17. PMID: 16293033.

9. Novotný T, Jeřábek J, Veselý K, Staffa $R$, Dvořák $M$, Cagaš J. Evaluation of a knitted polytetrafluoroethylene mesh placed intraperitoneally in a New Zealand white rabbit model. Surg Endosc. 2012 Jul;26(7):1884-91. PMID: 22219009.

10.Penttinen R, Grönroos JM. Mesh repair of common abdominal hernias: a review on experimental and clinical studies. Hernia. 2008 Aug;12(4):337-44. PMID: 18351432.

\section{Correspondence:}

Diego Paim Carvalho Garcia

Rua dos Timbiras, 3642 /sala 704

30140-062 Belo Horizonte - MG Brasil

Tel.: (55 31)3295-3411

diegopcg25@hotmail.com

Received: July 17, 2017

Review: Sept 18, 2017

Accepted: Oct 21, 2017
Conflict of interest: none

Financial source: none
${ }^{1}$ Research performed at Laboratory of Experimental Surgery, Department of Surgery, Medicine School, Universidade Federal de Minas Gerais (UFMG), Belo Horizonte-MG, Brazil. 